\title{
EDITORIAL
}

(O) NEPHROLOGY IN THE DEVELOPING WORLD

\section{The globalization of nephrology}

T he medical challenges faced by low-to-middle income countries (LMICs) are vast. Their populations are often disproportionately affected by a high burden of disease owing to a variety of factors including environmental exposures, malnutrition and unhealthy behaviours, exacerbated by a lack of resources and access to care. This cumulative, multifactorial burden is particularly true for kidney disease, whereby factors associated with poverty, such as low birth weight and exposure to infectious agents, are coupled with the often prohibitive costs associated with dialysis and transplantation. This focus issue of Nature Reviews Nephrology, published to coincide with the 2015 International Society of Nephrology (ISN) World Congress of Nephrology in Cape Town, explores some of the issues facing individuals with kidney disease in LMICs.

The latest report from the Global Burden of Disease Study highlights the scale of kidney disease as a cause of mortality worldwide (GBD 2013 Mortality and Causes of Death Collaborators. Lancet http://dx.doi.org/10.1016/ S0140-6736(14)61682-2). According to these data, overall mortality associated with chronic kidney disease (CKD) increased by nearly 37\% between 1990 and 2013. The effects of CKD on life expectancy were most notable in central Latin America; however, the study also highlighted regional variations in diseases that predispose to kidney disease. Ischaemic heart disease-a risk factor for CKD - was identified as the most common cause of mortality in many high-income countries, particularly in Western Europe and North America. By contrast, HIV/ AIDS-which was responsible for 1,341,000 deaths in 2013 - was the leading cause of early death across much of sub-Saharan Africa. High rates of mortality attributable to hepatitis C-induced cirrhosis were evident in Egypt and sickle cell disease was a major cause of decreased life expectancy in western sub-Saharan Africa.

Treatment of these diseases and their renal manifestations requires expert, specialized care that is often challenging in under-resourced settings; however, success stories, such as the widespread provision of combination antiretroviral therapy (cART) for HIV across socioeconomic boundaries is testament to the fact that universal access to essential care and treatment services is possible. Prolonged use of cART and the associated increased longevity of patients with HIV have led to new patterns of HIV-associated CKD. In this issue, Jeffrey Kopp and colleagues describe the epidemiology and mechanisms of $\mathrm{HIV}$-associated nephropathy as well as approaches to its diagnosis and treatment.

Similar to the trend seen for CKD, the age-standardized death rate associated with sickle cell disorders increased between 1990 and 2013. In their Review, Karl Nath and Robert Hebbel provide an overview of the diverse renal manifestations of sickle cell disease, their underlying pathogenetic mechanisms, and the unique challenges they present in terms of therapeutic options.

Patients with CKD are at increased risk of hepatitis C virus (HCV) infection and conversely, $\mathrm{HCV}$ infection can itself cause some forms of glomerulonephritis. Jose Morales and Fabrizio Fabrizi review the treatment options available to patients with HCV infection, including renal transplant recipients, and discuss the safe use of organs donated by infected individuals.

In all LMICs, issues of nutrition and birth weight prevail; nutritional deficiencies were responsible for 681,100 deaths in 2013. Malnutrition or overnutrition during early development can have lifelong effects on renal function. In their Review, Valerie Luyckx and Barry Brenner consider the impact of fetal and early life programming on the risks of hypertension and kidney disease in later life, and discuss the potential of nutritional interventions to reduce the global epidemic of these chronic diseases.

What other steps can be taken to reduce the burden of kidney disease in LMICs? Provision of training is essential to develop local nephrology expertise, and the ISN have made great progress in supporting medical education via their outreach programmes. Likewise, the ISN's ' 0 by 25 ' initiative aims to develop sustainable infrastructures within resource-poor countries with the ultimate goal of eliminating preventable deaths from acute kidney injury (AKI) by 2025. In line with this goal, the 'Saving Young Lives' project-a collaborative effort of the ISN, International Pediatric Nephrology Association, International Society for Peritoneal Dialysis and the Sustainable Kidney Care Foundation-aims to develop sustainable programmes for treating AKI in sub-Saharan Africa and South-East Asia. Two Perspectives articles in this issue highlight approaches to improve access to renal care in LMICs. Brian Remillard and colleagues describe their ongoing efforts to develop nephrology services in Haiti and highlight the role of partnerships in establishing a long-term commitment to nephrology education and care. Finally, Ikechi Okpechi, Charles Swanepoel and Francois Venter express their thoughts on how drug access can be improved in LMICs.

Together these articles provide an insight into the diverse issues facing the renal health of the developing world and the approaches by which nephrology care can be implemented on a global scale.

doi:10.1038/nrneph.2015.11

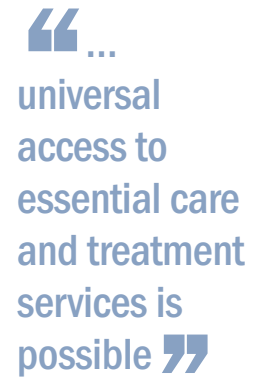

Susan J. Allison is the Chief Editor of Nature Reviews Nephrology.

Competing interests The author declares no competing interests. 